\title{
Improving the quality of production of gears in terms of errors in the profile of teeth in gear milling operations
}

\author{
Damir Safarov ${ }^{1, *}$ and Alexey Kondrashov ${ }^{1}$ \\ ${ }^{1}$ Naberezhnye Chelny institute (branch) of the Kazan (Volga) Federal University, 423812, \\ Naberezhnye Chelny, Mira Ave., 68/19, Russia
}

\begin{abstract}
The article describes the scheme for continual improvement of manufacturing quality spur gears in terms of error profiles of the side surfaces of gear teeth. The technique is universal and applicable to any operations of gear processing. The practical application of the technique is considered by the example of gear milling with a worm gear cutter. The errors of profile include metrological indicators of total error profile, the error direction and error of form profile. The scheme of analysis of the data consists of several steps - evaluation of indicators relative to the limit values, identification of the technological structure of the profile errors and expert analysis of the profile profilograms. The final expert analysis of profilograms allows to reveal the most probable operating negative technological factors leading to deviations of a profile from nominal position. The advantage of the quality improvement scheme is its high efficiency and effectiveness in comparison with traditional methods of statistical quality management, since it does not require the collection of significant amounts of statistical information.
\end{abstract}

\section{Introduction}

The key parameters that determine the position of the contact spot during the transmission of torque in the gear are the errors of the gear profiles. These parameters are provided at the finish gear grinding operation. Ensuring the profile parameters of the lateral surfaces of the teeth depends largely on the provision of their nominal values after the gear milling operation.

Error profile are the performance profiles:

- The total error of the profile F $\alpha$.

- The error of the direction of the profile FHa.

- Form error profile ffa

It also includes indicators of the longitudinal profile:

- The total error of the longitudinal profile $F \beta$.

- The error of the longitudinal direction of the profile FH $\beta$.

- The form error of the longitudinal profile ff $\beta$

\footnotetext{
* Corresponding author: Safarov-dt@mail.ru
} 
The main source of information about the quality indicators of the ring gear is the measurement protocols of specialized coordinate measuring centers with numerical control. Numerical values of errors are calculated by the computing center and are given in tabular forms below the field of profilograms.

Manufacturers of engineering products for the purpose of improving the quality of products are widely used methods of quality management. These include probabilistic and statistical methods and control maps. The use of these standard tools requires the accumulation of a significant amount of information for the calculation of the distribution law indicators of the studied quality indicators, as well as the values of the control boundaries. The result of the use of standard quality management tools is a conclusion about the certification of the process.

In practice, the conclusion of the certification is only a signal to search for the causes of inconsistencies, but does not bring the engineering services of enterprises to the actual identification of operating in the process of negative technological factors that lead to deviations in the quality of manufactured products.

\section{Theoretical part}

To reduce the complexity of identifying the causes of inconsistencies, a scheme for analyzing these profile errors was developed. In contrast to the statistical methods of quality management for the application of the developed technique is sufficient data on the actual values of the error profiles of two or three protocols of measurement of manufactured gears. The data analysis scheme is shown in figure 1 .

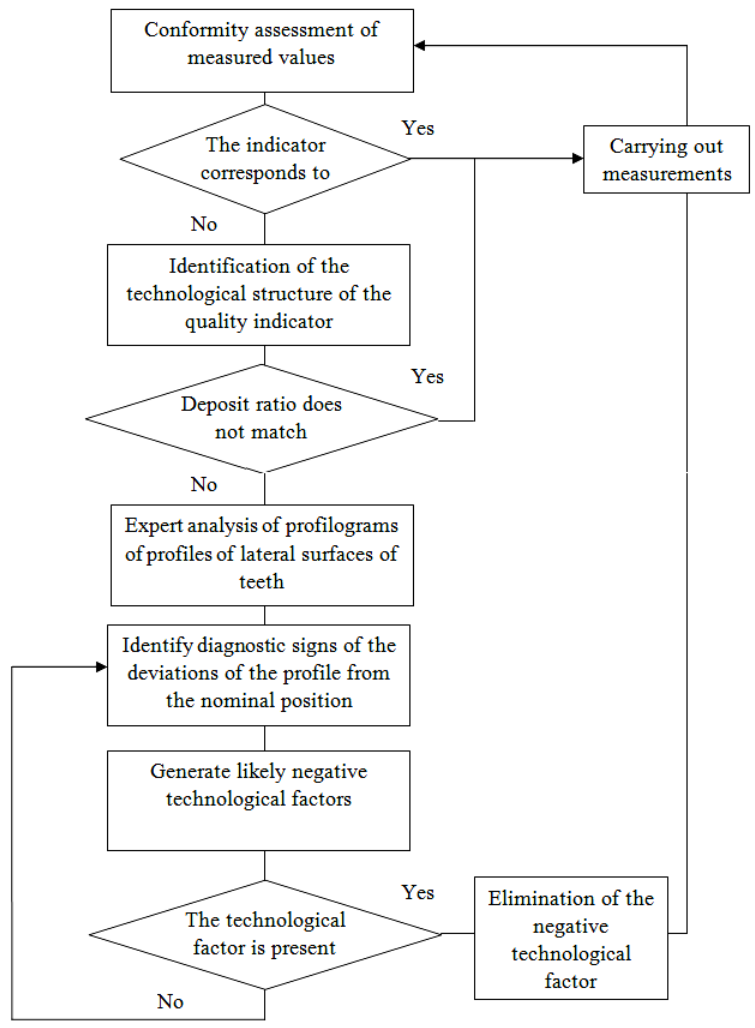

Fig. 1. The scheme of analysis of these errors profile to improve the quality of manufacture of gears. 
The diagram consists of the following sequential steps:

1. Estimates of indicators relative to the limit values established by the technological documentation. To ensure the greatest efficiency of the step, it is necessary to ensure the compliance of the investigated indicator in the technological operations of the route of the gear manufacture.

2. Identification of the technological structure of profile errors. The technological structure of the indicator determines the nesting of metrological indicators (figure 2).

On the lower level there is a form error of the tooth profile of ff $\alpha$ and $\mathrm{ff} \beta$ for these indicators higher level is the level to which belong the angular error profiles of the teeth of the crown $\mathrm{FH} \alpha$ and $\mathrm{FH} \beta$, the next level is the total error of the tooth profile $\mathrm{F} \alpha$ and $\mathrm{F} \beta$. The senior level is the level that owns the total error of the profiles of the lateral surface of the tooth. It can be calculated as the sum of total errors in the end and longitudinal section of the tooth of the ring gear $F \alpha \beta=F \alpha+F \beta$.

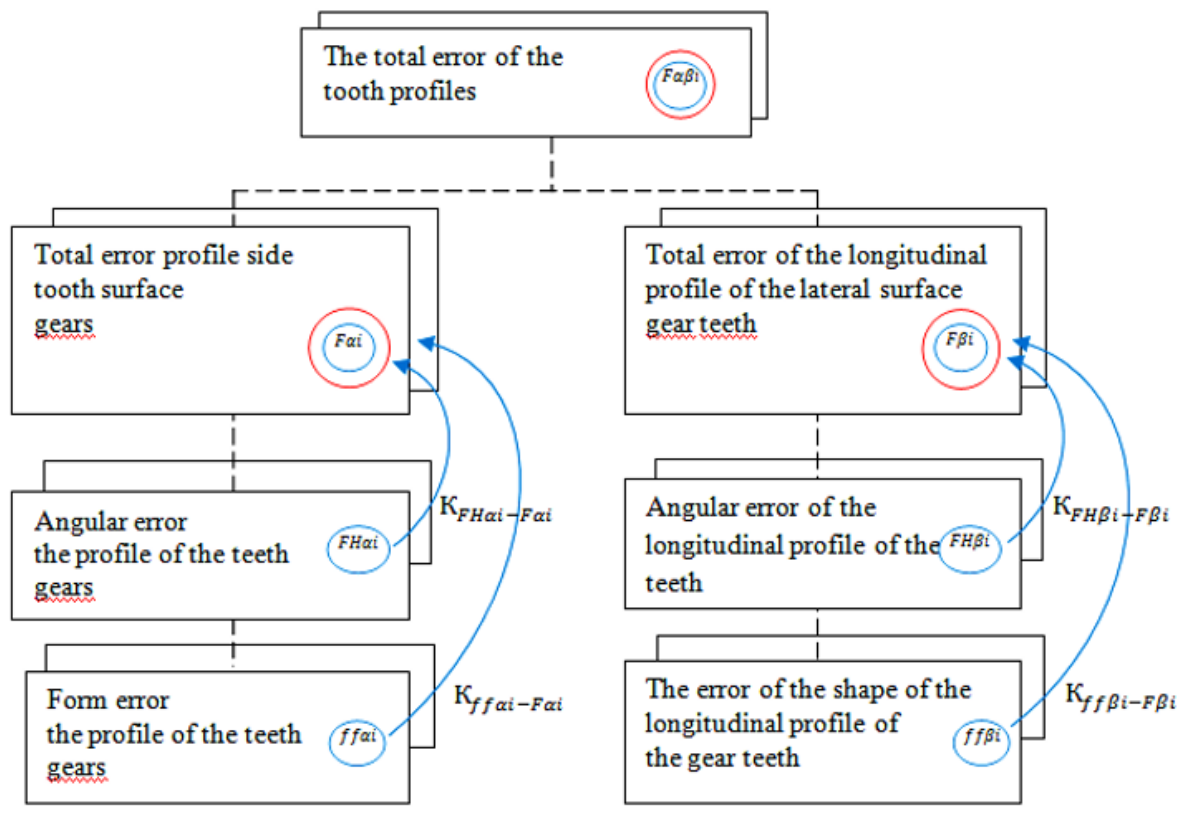

Fig. 2. Technological structure of the profile errors of the lateral surfaces of the gear teeth.

Technological structure is determined by calculation of coefficients of contribution of the indicators of lower metrological level indicator older technological level. Contribution factors are calculated for each metrological indicator of each level of the technological structure of the profile errors.

For example, for the errors of the longitudinal profile, the contribution of the errors of the angular direction of the longitudinal profile to the total error of the longitudinal profile can be calculated by the following formula:

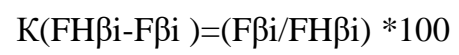

where $F \beta i$ - the total error of the longitudinal profile after the i-th technological operation $[\mu \mathrm{m}]$.

$\mathrm{KH} \beta \mathrm{i}$ - angular error of the direction of the longitudinal profile after the i-th technological operation $[\mu \mathrm{m}]$. 
The contribution of the longitudinal profile shape error to the total longitudinal profile error in the i-th technological operation [\%] is calculated by the following formula:

$$
K(\mathrm{ff} \beta \mathrm{i}-\mathrm{F} \beta \mathrm{i})=(\mathrm{F} \beta \mathrm{i} / \mathrm{ff} \beta \mathrm{i}) * 100
$$

where $\mathrm{F} \beta \mathrm{i}$ - the total error of the longitudinal profile after the i-th technological operation $[\mu \mathrm{m}]$.

$\mathrm{ff} \beta \mathrm{i}$ - accuracy of shape of the longitudinal profile after the i-th technological operation $[\mu \mathrm{m}]$.

The coefficients of the contribution of metrological indicators of other levels of the technological structure are the same way. In the numerator of the value of the indicator of the overlying level, in the denominator of the current. The limit values of the contribution of metrological indicators of the technological structure is established in accordance with the requirements of the standard [1].

3. Performing expert analysis of profilogram profiles is performed to identify negative technological factors leading to the discrepancies of the values of errors and high values of the coefficients of the contribution of metrological indicators in their technological structure.

The registered profile of a lateral surface which is a part of the Protocol of measurements of a gear ring contains information on the operating technological factors. The content of corrective actions can be detected by the presence of the profile of the side of the tooth diagnostic signs of deviation from its nominal position.

The cause of the diagnostic signs of the profile are known and strictly defined technological factors. Their finding is possible according to reference data, manufacturers of gear machining systems [2].

The authors have developed similar methods for other types of parts, based on the identification of diagnostic indicators of the quality of automotive components, which showed high efficiency of product improvement [3-9].

Also, factors and diagnostic features can be supplemented from a survey of the most experienced and qualified Adjusters gear cutting machines and the results of special production experiments. The found technological factors must be included in the PFMEA Protocol for the analysis of the technological operation.

The more extensive the database of diagnostic features of profilograms, the more effective will be the implementation of corrective and preventive actions to improve the quality of production of gears.

\section{Practical implementation}

Consider an example of the implementation of the proposed data processing scheme errors tooth profiles for processing helical gear in gear hobbing CNC operation. Processing of the toothed crown is performed by milling the worm gear cutter. 


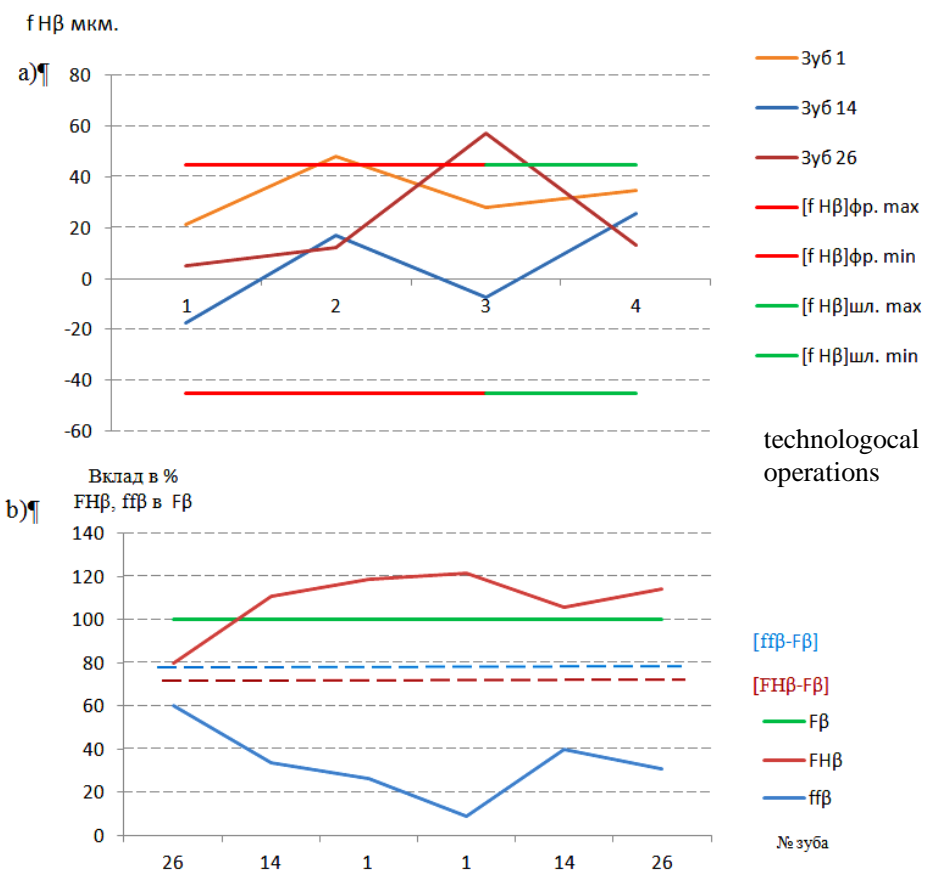

Fig. 3. Charts run the data processing circuit errors of the teeth a) Graph of change of error of the direction of profile on the technological path 1-gear hobbing CNC operation, 2-the operation of heat treatment 3 - integrated operation with CNC, 4 - gear grinding CNC operation, b) a graph of the ratio of the contribution of errors in the angular direction and form error of the profile.

To implement the first step of the developed method, three gears were measured after gear milling operation with CNC. According to the measurement protocols revealed considerable variation in terms of the error of the direction of the longitudinal profile F_H $\beta$. The values of the indicator are close to the upper third of both the lower and upper limit values, which can lead to potential inconsistencies of the machined gears.

As a result, additional measurements of the gears obtained values of errors and the graphs of the changes in the indices of the angular error for subsequent technological operations of manufacture of gear (1-hobbing operation, 2-heat operation 3-complex, CNC 3-grinding CNC operation). After thermal and complex CNC operation on individual gears there are inconsistencies (figure $3 \mathrm{a}$ ). Two of the three teeth have a profile error beyond the tolerance field. The scale of the indicator on the teeth is comparable to the value of the tolerance field.

According to the next step of the analysis of these errors, the actual technological structure of the error of the longitudinal direction of the profile is revealed. The graph of its change in the measured teeth of the toothed crown is shown in figure $3 \mathrm{~b}$. As we can see for all the measured teeth of the toothed crown on the right and left side, the coefficient of the index contribution to the total error of the profile significantly exceeds the coefficient values established by the standard [1]. To achieve the maximum effect of improving the quality of production of the gear, first of all, it is necessary to reduce the contribution of angular error to the total error of the profile and only then can various statistical methods of quality management be used.

The results of the final step of the search for negative factors of the process are given in table 1 . The first column of the first row of the table shows the profilogram of the studied teeth of the gear, the second diagnostic signs of profile deviations from the nominal position. 
Each diagnostic feature is a consequence of a strictly defined set of technological factors. In this case, in terms $\mathrm{F}_{-} \mathrm{H} \beta$ we have the existence of different angular direction longitudinal profiles of the different teeth of the ring gear.

Table 1 Profilogram and diagnostic signs of profile deviations from the nominal position.

\begin{tabular}{|l|l|l|}
\hline Profilogram Protocol parameters measuring accuracy \\
of the ring gear
\end{tabular}

According to the content requirements of the manufacturer's manual for gear cutting machines $\mathrm{f}$. Reishauer [2] the cause of this symptom is a multidirectional angular direction profiles of the different teeth can be any crooked areas axis unclamp the mandrel in different angular positions during grinding of the gear teeth to the direction of feed of hob. The deviation can be eliminated by additional adjustment of the mandrel. The presence of problems with the alignment of the position of the mandrel requires changing the support elements in order to reduce the error of its basing and reconciliation in the process of commissioning.

The value of the indicator $\mathrm{F} \alpha$ as a result of the measurement was close to the upper limit of the tolerance field, so for this indicator the profilograms profile was identified diagnostic markers and identified a likely active in the cutting process technological factors (table 1, row 2).

The developed data processing scheme allows for the most rapid and effective improvement of the quality of production immediately eliminating the negative technological factors that act in the process of manufacturing gears. The introduction of the technique on both preliminary and finishing operations of gear processing significantly improves the quality of production of gears with a significant reduction in the complexity of the improvement process. 


\section{Summary}

The technological structure of metrological indicators is the basis for the metrological examination of the correctness of the purpose of tolerances on the quality of cylindrical straight-toothed and helical gears design and technological documentation of the current production.

The developed technique of quality improvement gives the maximum effect at the stage of preparation of production at development of production of new gears when debugging of technological process is carried out. Only after providing the nominal technological structure of the tooth profile errors and eliminating the presence of diagnostic signs of profile deviations from their nominal position, it is possible to apply standard methods of product quality control.

\section{References}

1. GOST ISO 1328-1-2017 Gears and cylindrical. ISO system. Classification of tolerances on the lateral surfaces of the teeth. Part 1. Definitions and permissible values of deviations on the lateral surfaces of the teeth of the gear (Moscow STANDARTINFORM 42 p. 2017)

2. J.F. Delavy, J Cadisch, W Thyssen, P Schacke, R Schwaighofer, Шлифование зубчатых колес, (Reishauer AG, 121 р. 1993)

3. D.T. Safarov, A.G. Kondrashov Technique for monitoring the indicators of the total contact spot of spur gears of the truck's differential gear, IOP Conference Series: Materials Science and Engineering, Vol. 457 No 1, 19 December 2018, article \# 012014 (2018)

4. D.T. Safarov, A.G. Kondrashov, Improving the processing accuracy of the valve seats of internal combustion engines using diagnostic measurements, Journal of Physics: Conference Series, Volume 1050, Issue 1, 26 July 2018, article \# 012075

5. S.V. Kasjanov, A.G. Kondrashov, D.T. Safarov, Regulation of Geometrical Parameters Deviations of Automotive Components, Parts through Diagnostic Measurements Organization Procedia Engineering, Volume 206, pp. 1508-1514 (2017)

6. S.V. Kas'yanov, D.T. Safarov, Diagnosis of technical state of equipment and tools according to indices of technological accuracy. Avtomobil'naya Promyshlennost, Issue 5, pp. 24-28 (2004)

7. A.G. Kondrashov, S.V. Kasyanov, D.T. Safarov and A.V. Kuznetsova, Diagnostic measurements of geometrical parameters of spatial and difficult details of autocomponents by one-coordinate altimeter Control, Diagnostics, Issue 8, pp. 60-64 (2013)

8. A.G. Kondrashov, D.T. Safarov, Prediction of accuracy when handling by cutting News of higher educational institutions, Mechanical engineering, Issue 12, pp. 63-69 (2014) 